\title{
Finding Critical Nodes for Inhibiting Diffusion of Complex Contagions in Social Networks
}

\author{
Chris J. Kuhlman ${ }^{1}$, V.S. Anil Kumar ${ }^{1}$, Madhav V. Marathe ${ }^{1}$, \\ S.S. Ravi ${ }^{2}$, and Daniel J. Rosenkrantz ${ }^{2}$ \\ 1 Virginia Bioinformatics Institute, Virginia Tech, Blacksburg, VA 24061, USA \\ \{ckuhlman, akumar, mmarathe\}@vbi.vt.edu \\ 2 Computer Science Department, University at Albany - SUNY, \\ Albany, NY 12222, USA \\ $\{$ ravi,djr\}@cs.albany.edu
}

\begin{abstract}
We study the problem of inhibiting diffusion of complex contagions such as rumors, undesirable fads and mob behavior in social networks by removing a small number of nodes (called critical nodes) from the network. We show that, in general, for any $\rho \geq 1$, even obtaining a $\rho$-approximate solution to these problems is NP-hard. We develop efficient heuristics for these problems and carry out an empirical study of their performance on three well known social networks, namely epinions, wikipedia and slashdot. Our results show that the heuristics perform well on the three social networks.
\end{abstract}

\section{Introduction and Motivation}

Analyzing social networks has become an important research topic in data mining (e.g. [31, 9, 20, 21, 7, 32 ). With respect to diffusion in social networks, researchers have studied the propagation of favorite photographs in a Flickr network [6], the spread of information [16, 23] via Internet communication, and the effects of online purchase recommendations [26], to name a few. In some instances, models of diffusion are combined with data mining to predict social phenomena (e.g., product marketing [9, 31] and trust propagation [17]).

Here we are interested in the diffusion of a particular class of contagions, namely complex contagions. As stated by Centola and Macy [5], "Complex contagions require social affirmation from multiple sources." That is, a person acquires a social contagion through interaction with $t>1$ other individuals, as opposed to a single individual (i.e., $t=1$ ); the latter is called a simple contagion.

As described by Granovetter [15], the idea of complex contagions dates back to the 1960's, and more current studies are referenced in [5,11. Such phenomena include diffusion of innovations, rumors, worker strikes, educational attainment, fashion, and social movements. For example, in strikes, mob violence, and political upheavals, individuals can be reluctant to participate for fear of reprisals to themselves and their families. It is safer to wait for a critical mass of people to commit before committing oneself. Researchers have used data mining 
techniques to study the propagation of complex contagions such as online DVD purchases [26] and teenage smoking initiation [19]. As discussed by Easley and Kleinberg [11, complex contagion is also closely related to Coordination Games. Motivation for our work came partially from recent quantitative work [5] showing that simple contagions and complex contagions can differ significantly in behavior. Further, it is well known [14 that weak edges play a dominant role in spreading a simple contagion between clusters within a population, thereby dictating whether or not a contagion will reach a large segment of a population. However, for complex contagions, this effect is greatly diminished [5] because chances are remote that multiple members, who are themselves connected within a group, are each linked to multiple members of another group.

The focus of our work is inhibiting the diffusion of complex contagions such as rumors, undesirable fads, and mob behavior in social networks. In our formulation, the goal is to minimize the spread of a contagion by removing a small number of nodes, called critical nodes, from the network. Other formulations of this problem have been considered in the literature for simple contagions (e.g. [18]). We will discuss the differences between our work and that reported in other references in Section 3. Applications of finding critical nodes in a network include thwarting the spread of sensitive information that has been leaked [7, disrupting communication among adversaries [1, marketing to counteract the advertising of a competing product [31,9], calming a mob [15], and changing people's opinions 10 .

We present both theoretical and empirical results. (A more technical summary of our results is given in Section 3) On the theoretical side, we show that for two versions of the problem, even obtaining efficient approximations is NPhard. These results motivate the development and evaluation of heuristics that work well in practice. We develop two efficient heuristics for finding critical sets and empirically evaluate their performance on three well known social networks, namely epinions, wikipedia and slashdot.

This paper is organized as follows. Section 2 describes the model employed in this work and presents the formal problem statement. Section 3 contains related work and a summary of results. Theoretical results are provided in Section 4 Two heuristics are described in Section 5 and are evaluated against three social networks in Section 6] Directions for future work are provided in Section 7 .

\section{Dynamical System Model and Problem Formulation}

\subsection{System Model and Associated Definitions}

We model the propagation of complex contagions over a social network using discrete dynamical systems [2,24]. We begin with the necessary definitions.

Let $\mathbb{B}$ denote the Boolean domain $\{0,1\}$. A Synchronous Dynamical System $(\mathrm{SyDS}) \mathcal{S}$ over $\mathbb{B}$ is specified as a pair $\mathcal{S}=(G, \mathcal{F})$, where

(a) $G(V, E)$, an undirected graph with $n$ nodes, represents the underlying social network over which the contagion propagates, and 


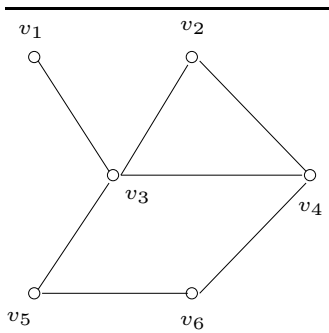

Initial Configuration:

$(1,1,0,0,0,0)$

Configuration at time $1:(1,1,1,0,0,0)$

Configuration at time $2:(1,1,1,1,0,0)$

Note: Each configuration has the form $\left(s_{1}, s_{2}, s_{3}, s_{4}, s_{5}, s_{6}\right)$, where $s_{i}$ is the state of node $v_{i}, 1 \leq i \leq 6$. The configuration at time 2 is a fixed point.

Fig. 1. An example of a synchronous dynamical system

(b) $\mathcal{F}=\left\{f_{1}, f_{2}, \ldots, f_{n}\right\}$ is a collection of functions in the system, with $f_{i}$ denoting the local transition function associated with node $v_{i}, 1 \leq i \leq n$.

Each function $f_{i}$ specifies the local interaction between node $v_{i}$ and its neighbors in $G$. We note that each node of $G$ has a state value from $\mathbb{B}$. To encompass various types of social contagions as described in Section 1 nodes in state 0 (1) are said to be unaffected (affected). In the case of information flow, an affected node could be one that has received the information. It is assumed that once a node reaches state 1 , it cannot return to state 0 . A discrete dynamical system with this property is referred to as a ratcheted dynamical system [24].

We can now formally describe the local interaction functions. The inputs to function $f_{i}$ are the state of $v_{i}$ and those of the neighbors of $v_{i}$ in $G$; function $f_{i}$ maps each combination of inputs to a value in $\mathbb{B}$. For the propagation of contagions in social networks, it is appropriate to model each function $f_{i}(1 \leq i \leq n)$ as a $t_{i}$-threshold function [12,7, 10, 4, 5, 20,22, for an appropriate nonnegative integer $t_{i}$. Such a threshold function (taking into account the ratcheted nature of the dynamical system) is defined as follows: (a) If the state of $v_{i}$ is 1 , then $f_{i}$ is 1 , regardless of the values of the other inputs to $f_{i}$, and (b) If the state of $v_{i}$ is 0 , then $f_{i}$ is 1 if at least $t_{i}$ of the inputs are 1 ; otherwise, $f_{i}$ is 0 .

A configuration $\mathcal{C}$ of a SyDS at any time is an $n$-vector $\left(s_{1}, s_{2}, \ldots, s_{n}\right)$, where $s_{i} \in \mathbb{B}$ is the value of the state of node $v_{i}(1 \leq i \leq n)$. A single SyDS transition from one configuration to another is implemented by using all states $s_{i}$ at time $j$ for the computation of the next states at time $j+1$. Thus, in a SyDS, nodes update their states synchronously. Other update disciplines (e.g. sequential updates) for discrete dynamical systems have also been studied [2] A configuration $\mathcal{C}$ is called a fixed point if the successor of $\mathcal{C}$ is $\mathcal{C}$ itself.

Example: Consider the graph shown in Figure 1. Suppose the local interaction function at each node is the 2-threshold function. Initially, $v_{1}$ and $v_{2}$ are in state 1 and all other nodes are in state 0 . During the first time step, the state of node $v_{3}$ changes to 1 since two of its neighbors (namely $v_{1}$ and $v_{2}$ ) are in state 1 ; the states of other nodes remain the same. In the second time step, the state of node $v_{4}$ changes to 1 since two of its neighbors (namely $v_{2}$ and $v_{3}$ ) are in state 1 ; 
again the states of the other nodes remain the same. The resulting configuration $(1,1,1,1,0,0)$ is a fixed point for this system.

The SyDS in the above example reached a fixed point. This is not a coincidence. The following general result (which holds for any ratcheted dynamical system over $\mathbb{B})$ is shown in $[24]$.

Theorem 1. Every ratcheted SyDS over $\mathbb{B}$ reaches a fixed point in at most $n$ transitions, where $n$ is the number of nodes in the underlying graph.

\subsection{Problem Formulation}

For simplicity, statements of problems and results in this paper use terminology from the context of information propagation in social networks, such as that for social unrest in a group; these can be easily extended to other contagions.

Suppose we have a social network in which some nodes are initially affected. In the absence of any action to contain the unrest, it may spread to a large part of the population. Decision-makers must decide on suitable actions to inhibit information spread, such as quarantining a subset of people, subject to resource constraints and societal pressures (e.g., quarantining too many people may fuel unrest or it may be cost prohibitive to apprehend particular individuals).

We assume that people who are as yet unaffected can be quarantined or isolated. Under the dynamical system model, quarantining a person is represented by removing the corresponding node (and all the edges incident on that node) from the graph. Equivalently, removing a node $v$ corresponds to changing the local transition function at $v$ so that $v$ 's state remains 0 for all combinations of input values. The goal of isolation is to minimize the number of new affected nodes that occur over time until the system reaches a fixed point (when no additional nodes can be affected). We use the term critical set to refer to the set of nodes removed from the graph to reduce the number of newly affected nodes. Recall that resource constraints impose a budget constraint on the size of the critical set. We can now provide a precise statement of the problem of finding critical sets. (This problem was first formulated in [12 for the case where each node computes a 1-threshold function.)

\section{Small Critical Set to Minimize New Affected Nodes (SCS-MNA)}

Given: A social network represented by the $\operatorname{SyDS} \mathcal{S}=(G(V, E), \mathcal{F})$ over $\mathbb{B}$, with each function $f \in \mathcal{F}$ being a threshold function; the set $I\left(n_{s}=|I|\right)$ of nodes which are initially in state 1 ; an upper bound $\beta$ on the size of the critical set.

Requirement: A critical set $C$ (i.e., $C \subseteq V-I$ ) such that $|C| \leq \beta$ and among all subsets of $V-I$ of size at most $\beta$, the removal of $C$ from $G$ leads to the smallest number of new affected nodes.

An alternative formulation, where the objective is to maximize the number of people who are not affected, can also be considered. We use the name "Small Critical Set to Maximize Unaffected Nodes" for this problem and abbreviate it as SCS-MUN. Clearly, any optimal solution for SCS-MUN is also an optimal 
solution for SCS-MNA. Our results in Section 4 provide an indication of the difficulties in obtaining provably good approximation algorithms for either version of the problem. So, our focus is on devising heuristics that work well in practice.

\subsection{Additional Terminology}

Here, we present some terminology used in the later sections of this paper. The term " $t$-threshold system" is used to denote a SyDS in which each local transition function is the $t$-threshold function for some integer $t \geq 0$. (The value of $t$ is the same for all nodes of the system.)

Let $\mathcal{S}=(G(V, E), \mathcal{F})$ be a SyDS and let $I \subseteq V$ denote the set of nodes whose initial state is 1 . We say that a node $v \in V-I$ is salvageable if there is a critical set $C \subseteq V-I$ whose removal ensures that $v$ remains in state 0 when the modified SyDS (i.e., the SyDS obtained by removing $C$ ) reaches a fixed point. Otherwise, $v$ is called an unsalvageable node. Thus, in any SyDS, only salvageable nodes can possibly be saved from becoming affected.

We also need some terminology with respect to approximation algorithms for optimization problems [13. For any $\rho \geq 1$, a $\rho$-approximation for an optimization problem is an efficient algorithm that produces a solution which is within a factor of $\rho$ of the optimal value for all instances of the problem. Such an approximation algorithm is also said to provide a performance guarantee of $\rho$. Clearly, the smaller the value of $\rho$, the better is the performance of the approximation algorithm.

The following terms are used in describing empirical results of Section 6. A cascade occurs when diffusion starts from a set of seed nodes (set $I$ ) and $95 \%$ or more of nodes that can be affected are affected. Halt means that a set of critical nodes will stop the diffusion process, thus preventing a cascade. A delay means that the set of critical nodes will increase the time at which the peak number of newly affected nodes occurs, but will not necessarily halt diffusion.

\section{Summary of Results and Related Work}

Our main results can be summarized as follows.

(a) We show that for any $t \geq 2$ and any $\rho \geq 1$, it is $\mathbf{N P}$-hard to obtain a $\rho$-approximation for either the SCS-MNA or the SCS-MUN problem for $t$ threshold systems. (The result holds even when $\rho$ is a function of the form $n^{\delta}$, where $\delta<1$ is a constant and $n$ is the number of nodes in the network.)

(b) We show that the problem of saving all salvageable nodes (SCS-SASN) can be solved in linear time for 1-threshold systems and that the required critical set is unique. In contrast, we show that the problem is NP-hard for $t$ threshold systems for any $t \geq 2$. We also develop an $O(\log n)$-approximation algorithm for this problem, where $n$ is the number of nodes in the network.

(c) We develop two intuitively appealing heuristics for the SCS-MNA problem and carry out an empirical study of their performance on three social 
networks, namely epinions, wikipedia and slashdot. Our experimental results show that in many cases, the two heuristics are similar in their ability to delay and halt the diffusion process. In general, one of the heuristics runs faster but there are cases where the other heuristic is more effective in inhibiting diffusion.

Related work on finding critical sets has been confined to threshold $t=1$. Further, the focus is on selecting critical nodes to inhibit diffusion starting from a small random set $I$ of initially infected (or seed) nodes. Our approach, in contrast, is focused on $t \geq 2$ and our heuristics compute a critical set for any specified set of seed nodes. Critical nodes are called "blockers" in [18. They examine dynamic networks and use a probabilistic diffusion model with threshold $=1$. They rely on graph metrics such as degree, diameter, and betweenness to identify critical nodes. In [7], the largest eigenvalue of the adjacency matrix of a graph is used to identify a node that causes the maximum decrease in the epidemic threshold. Vaccinating such a node reduces the likelihood of a large outbreak. A variety of network-based candidate measures for identifying critical nodes under threshold 1 conditions are described in 3]; however, the applications are confined to small networks. Hubs, or high degree nodes in scale free networks, have also been investigated as critical nodes, using mean field theory, in 8. Reference 12 presents an approximation algorithm for the problem of minimizing the number of new affected nodes for 1-threshold systems. Reference 28] considers the problem of detecting cascades in networks and develops submodularity-based algorithms to determine the size of the affected population before a cascade is detected.

\section{Theoretical Results for the Critical Set Problem}

In this section, we first present complexity results for finding critical sets. We also present results that show a significant difference between 1-threshold systems and $t$-threshold systems where $t \geq 2$. Owing to space limitations, we have omitted proofs of these results; they can be found in [25].

\subsection{Complexity Results}

As mentioned earlier, the SCS-MNA problem was shown to be NP-complete in 12. for the case when each node has a 1-threshold function. We now extend that result, and include a result for the SCS-MUN problem, to show that even obtaining $\rho$-approximate solutions is NP-hard for systems in which each node computes the $t$-threshold function for any $t \geq 2$.

Theorem 2. Assuming that the bound $\beta$ on the size of the critical set cannot be violated, for any $\rho \geq 1$ and any $t \geq 2$, there is no polynomial time $\rho$-approximation algorithm for either the SCS-MNA problem or the SCS-MUN problem for $t$-threshold systems, unless $\boldsymbol{P}=\boldsymbol{N P}$.

Proof: See [25]. 


\subsection{Critical Sets for Saving All Salvageable Nodes}

Recall from Section 2.3 that a node $v$ of a SyDS is salvageable if there is a critical set whose removal ensures that $v$ will not be affected. We now consider the following problem which deals with saving all salvageable nodes.

\section{Small Critical Set to Save All Salvageable Nodes (SCS-SASN):}

Given: A social network represented by the $\operatorname{SyDS} \mathcal{S}=(G(V, E), \mathcal{F})$ over $\mathbb{B}$, with each function $f \in \mathcal{F}$ being a threshold function; the set $I$ of nodes which are initially in state 1 .

Requirement: A critical set $C$ (i.e., $C \subseteq V-I$ ) of minimum cardinality whose removal ensures that all salvageable nodes are saved from being affected.

For the above problem, we present results that show a significant difference between 1-threshold systems and $t$-threshold systems where $t \geq 2$.

Theorem 3. Let $\mathcal{S}=(G(V, E), \mathcal{F})$ be a 1-threshold SyDS. The SCS-SASN problem for $\mathcal{S}$ can be solved in $O(|V|+|E|)$ time. Moreover, the solution is unique.

Proof: See 25].

The next result concerns the SCS-SASN problem for $t$-threshold systems, where $t \geq 2$.

Theorem 4. The SCS-SASN problem is $\mathbf{N P}$-hard for $t$-threshold systems, where $t \geq 2$. However, there is an $O(\log n)$-approximation algorithm for this problem, where $n$ is the number of nodes in the network.

Proof: See 25].

\section{Heuristics for Finding Small Critical Sets}

\subsection{Overview}

As can be seen from the complexity results presented in Section 4 it is difficult to develop heuristics with provably good performance guarantees for the SCSMNA and SCS-MUN problems. So, we focus on the development of heuristics that work well in practice for one of these problems, namely SCS-MNA. In this section, we present two such heuristics that are evaluated in Section 6. The first heuristic uses a set cover computation. The second heuristic relies on a potential function, which provides an indication of a node's ability to affect other nodes.

\subsection{Covering-Based Heuristic}

Given a SyDS $\mathcal{S}=(G(V, E), \mathcal{F})$ and the set $I \subseteq V$ of nodes whose initial state is 1 , one can compute the set $S_{j} \subseteq V$ of nodes that change to state 1 at the $j^{\text {th }}$ time step, $1 \leq j \leq \ell$, for some suitable $\ell \leq|V|$. The covering-based heuristic (CBH) chooses a critical set $C$ as a subset of $S_{j}$ for some suitable $j$. The intuitive reason for doing this is that each node $w$ in $S_{j+1}$ has at least one neighbor $v$ in 
Input: A SyDS $\mathcal{S}=(G(V, E), \mathcal{F})$, the set $I \subseteq V$ of nodes whose initial state is 1 , the upper bound $\beta$ on the size of the critical set and the number of initial simulation steps $\ell \leq|V|$.

Output: A critical set $C \subseteq V-I$ whose removal leads to a small number of new affected nodes.

\section{Steps:}

1. Simulate the system for $\ell$ time steps and determine sets $S_{1}, S_{2}, \ldots, S_{\ell}$, where $S_{j}$ is the set of newly affected nodes at time $j, 1 \leq j \leq \ell$.

2 . if any set $S_{j}$ has at most $\beta$ nodes, then output such a set as the critical set and stop. (When there are ties, choose the set $S_{j}$ with the smallest value of $j$.)

3. Comment: Here, all the $S_{j}$ 's have $\beta+1$ or more nodes.

(i) for $j=1$ to $\ell-1$ do

(a) For each node $v_{j} \in S_{j}$, construct the set $\Gamma_{j}$ which consists of all the neighbors of $v_{j}$ in $S_{j+1}$ that can be prevented from becoming affected by removing $v_{j}$. Let $\Gamma$ denote the collection of all the sets constructed.

(b) Use a greedy approach to find a subcollection $\Gamma^{\prime}$ of $\Gamma$ containing at most $\beta$ sets so as to cover as many elements of $S_{j+1}$ as possible.

(c) Let the critical set $C$ consist of the nodes of $S_{j}$ corresponding to the elements of $\Gamma^{\prime}$.

(ii) Among all the critical sets $C$ considered in Step 3(i)(c), output the one $C$ that occurs earliest in time that covers all nodes of $\Gamma$, and if no such $C$ exists, output the earliest $C$ such that $\left|S_{j}\right|-|C|$ is minimum.

Fig. 2. Details of the covering-based heuristic

$S_{j}$. (Otherwise, $w$ would have changed to 1 in an earlier time step.) Therefore, if a suitable subset of $S_{j}$ can be chosen so that none of the nodes in $S_{j+1}$ changes to 1 during the $(j+1)^{\text {st }}$ time step, the contagion cannot spread beyond $S_{j}$. In general, when nodes have thresholds $\geq 2$, the problem of choosing at most $\beta$ nodes from $S_{j}$ to prevent a maximum number of nodes in $S_{j+1}$ from changing to 1 is also NP-hard. (This result can be proven in a manner similar to that of Theorem 2.) Therefore, we use a greedy approach for this step. In each iteration, this approach chooses a node from $S_{j}$ that saves the largest number of nodes in $S_{j+1}$ from becoming affected. The greedy approach is repeated for each $j$, $1 \leq j \leq \ell-1$. The steps of the covering-based heuristic are shown in Figure 2, In Step 2, when two or more sets have $\beta$ or fewer nodes, we choose the one that corresponds to an earlier time step since such a choice can save more nodes from becoming affected.

\subsection{Potential-Based Heuristic}

The idea of the potential-based heuristic $(\mathrm{PBH})$ is to assign a potential to each node $v$ depending on how early $v$ is affected and how many nodes it can affect 
Input: $\operatorname{A} \operatorname{SyDS} \mathcal{S}=(G(V, E), \mathcal{F})$, the set $I \subseteq V$ of nodes whose initial state is 1 , the upper bound $\beta$ on the size of the critical set.

Output: A critical set $C \subseteq V-I$ whose removal leads to a small number of new affected nodes.

\section{Steps:}

1. Simulate the system $\mathcal{S}$ and determine sets $S_{1}, S_{2}, \ldots, S_{T}$, where $T$ is the time step at which $\mathcal{S}$ reaches a fixed point and $S_{j}$ is the set of newly affected nodes at time $j, 1 \leq j \leq T$.

2. for each node $x \in S_{T}$ do $P[x]=0$.

3. for $j=T-1$ downto 1 do

for each node $x \in S_{j}$ do

(a) Find $N_{j+1}[x]$ and let $P[x]=\left|N_{j+1}[x]\right|$.

(b) for each node $y \in N_{j+1}[x]$ do

$$
P[x]=P[x]+P[y]
$$

(d) Set $P[x]=(T-j)^{2} P[x]$.

4. Let the critical set $C$ contain $\beta$ nodes with the highest potential among all the nodes. (Break ties arbitrarily.) Output $C$.

Fig. 3. Details of the potential-based heuristic

later. Nodes with larger potential values are more desirable for inclusion in the critical set. While CBH chooses a critical set from one of the $S_{j}$ sets, the potential based approach may select nodes in a more global fashion from the whole graph. One can obtain different versions of $\mathrm{PBH}$ by choosing different potential functions. We have chosen one that is easy to compute. Details of PBH are shown in Figure 3 .

We assume that set $S_{j}$ of newly affected nodes at time $j$ has been computed for each $j, 1 \leq j \leq T$, where $T$ is the time at which the system reaches a fixed point. For any node $x \in S_{j}$, let $N_{j+1}[x]$ denote the set of nodes in $S_{j+1}$ which are adjacent to $x$ in $G$. The potential $P[x]$ of a node $x$ is computed as follows:

(a) For each node $x$ in $S_{T}, P[x]=0$. (Justification: There is no diffusion beyond level $T$. So, it is not useful to include nodes from $S_{T}$ in the critical set.) (b) For each node $x$ in level $j, 1 \leq j \leq T-1$,

$$
P[x]=(T-j)^{2}\left[\left|N_{j+1}[x]\right|+\sum_{y \in N_{j+1}[x]} P[y]\right]
$$

(Justification: The term $(T-j)^{2}$ decreases as $j$ increases. Thus, higher potentials are assigned to nodes that are affected earlier. The term $\left|N_{j+1}[x]\right|$ gives more weight to nodes that have a large number of neighbors in the next level.) 


\section{Empirical Evaluation of Heuristics}

\subsection{Networks, Study Parameters and Test Procedures}

Table1provides selected features of three social networks used in this study. We assume all edges are undirected to foster greater diffusion and thereby test more stringently the heuristics. The degree and clustering coefficient1 distributions for the three networks are given elsewhere [25].

Table 1. Three networks [30,29,27] and selected characteristics

\begin{tabular}{|c|c|c|c|c|}
\hline Network & $\begin{array}{c}\text { Number } \\
\text { of Nodes }\end{array}$ & $\begin{array}{c}\text { Number } \\
\text { of Edges }\end{array}$ & $\begin{array}{c}\text { Average } \\
\text { Degree }\end{array}$ & $\begin{array}{c}\text { Average Clustering } \\
\text { Coefficient }\end{array}$ \\
\hline epinions & 75879 & 405740 & 10.7 & 0.138 \\
\hline wikipedia & 7115 & 100762 & 28.3 & 0.141 \\
\hline slashdot & 77360 & 469180 & 12.1 & 0.0555 \\
\hline
\end{tabular}

Table 2 lists the parameters and values used in the parametric study with the networks to evaluate the two heuristics. For a given value of number of seeds $n_{s}, 100$ sets of size $n_{s}$ were determined from each network to provide a range of cases for testing the heuristics. Each seed node was taken from a 20-core, a subgraph in which each node has a degree of at least 20 . The 20 -core was a good compromise between selecting high-degree nodes, and having a sufficiently large pool of nodes to choose from so that sets of seeds overlapped little. Moreover, every seed node in a set is adjacent to at least one other seed node, so the seeds were "clumped," in order to foster diffusion. Thus, the test cases utilized two means, namely seeding of high-degree nodes and clumping the seed nodes, to foster diffusion and hence tax the heuristics.

Table 2. Parameters and values of parametric study

\begin{tabular}{|c|c|c|c|}
\hline Thresholds, $t$ & $\begin{array}{c}\text { Numbers } \\
\text { of Seeds, } n_{s}\end{array}$ & $\begin{array}{c}\text { Budgets of } \\
\text { Critical Nodes, } \beta\end{array}$ & $\begin{array}{c}\text { Number of } \\
\text { Replicates }\end{array}$ \\
\hline $2,3,5$ & $2,3,5,10,20$ & $5,10,20,50,100,500$ & 100 \\
\hline
\end{tabular}

The test plan consists of running simulations of 100 iterations each ( 1 iteration for each seed node set) on the three networks for all combinations of $t, n_{s}$, and $\beta$. All nodes except seed nodes are initially in the unaffected state. Our simulator outputs for each node the time at which it is affected. The heuristics use this as input data and calculate one set of $\beta$ critical nodes for each iteration. The simulations are then repeated, but now they include the critical nodes, so that

\footnotetext{
${ }^{1}$ For a node $v$ in a graph $G$, the clustering coefficient $c_{v}$ is defined as follows. Let $N(v)$ denote the set of nodes adjacent to $v$. Then, $c_{v}$ is the ratio of the number of edges in the subgraph induced on $N(v)$ to the number of edges in a complete graph on $N(v)$.
} 
the decrease in the total number of affected nodes caused by a critical set can be quantified. Heuristic computations and simulations were performed on a 96-node cluster (2 processors/node; 4 cores/processor), with $3 \mathrm{GHz}$ Intel Xeon cores and $2 \mathrm{MB}$ memory per core.

\subsection{Results}

A summary of our main experimental findings is as follows. The discussion uses some of the terminology (namely, cascade, halt and delay) from Section 2.3

\section{Structural results}

(a) Critical node sets either halt diffusion with very small affected set sizes or do not prevent a cascade; thus, critical nodes generate phase transitions (unless all iterations halt the diffusion).

(b) The fraction of iterations cascading behaves as $(1 / \beta)$ for $n_{s} \leq 5$, so to halt diffusion over all iterations can require $\beta \geq 500=100 n_{s}$. This is in part attributable to the stochastic nature of the seeding process. While a heuristic may be successful on average, there will be combinations of seed nodes that are particularly difficult to halt.

(c) In some cases, if diffusion is not halted, a delay in the time to reach the peak number of newly affected nodes can be achieved, thus providing a retarding effect. This is a consequence of computed critical nodes impeding initial diffusion near the start time, but being insufficient to halt the spread. For the deterministic diffusion of this study, it is virtually impossible to impede diffusion after time step 2 or 3 because by this time, too many nodes have been affected.

\section{Quality of solution}

(d) The heuristics perform far better than setting high-degree nodes critical, and setting random nodes critical ("null" condition).

(e) For $n_{s} \leq 5$ and $\beta \leq 50$, the two heuristics often give similar results, and do not always halt diffusion. For small numbers of seeds, $\mathrm{PBH}$, which is purposely biased toward selecting nodes affected early in the diffusion process, selects nodes at early times. $\mathrm{CBH}$ also seeks to halt at early times. Hence, both heuristics are trying to accomplish the same thing.

(f) However, when $\beta \geq 100$ nodes are required to stop diffusion because of a larger number of seeds, $\mathrm{CBH}$ is more effective in halting diffusion because it focuses critical nodes at one time step as explained below; hence there can be a tradeoff between speed of computation and effectiveness of the heuristics since $\mathrm{PBH}$ executes faster.

Figure 4 depicts the execution times for each heuristic for $\beta=5$. For the epinions network, Figure 4(a), these times translate into a maximum of roughly 1.5 hours for $\mathrm{CBH}$ to determine 100 sets of critical nodes, versus less than 5 minutes for PBH. For the wikipedia network, Figure 4(b) comparable execution 


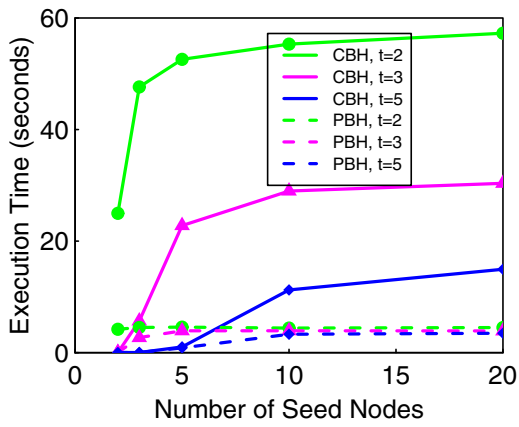

(a)

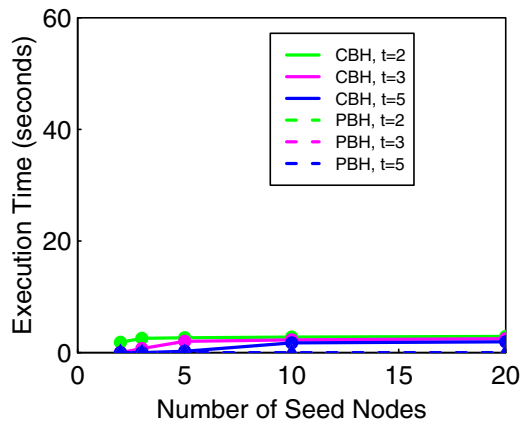

(b)

Fig. 4. Times for $\mathrm{CBH}$ and $\mathrm{PBH}$ to compute one set of critical nodes as a function of threshold and number of seeds for the (a) epinions network; (b) wikipedia network. Times are averages over 100 iterations.

times are observed when the number of nodes decreases by an order of magnitude. As described in Section 5, PBH evaluates every node once, whereas a node in $\mathrm{CBH}$ is often analyzed at many different time steps.

We now turn to evaluating the heuristics in halting and delaying diffusion by first comparing the heuristics with the heuristics of (1) randomly setting nodes critical ( $\mathrm{RCH})$, and (2) setting high-degree nodes critical ( $\mathrm{HCH})$. Table 3 summarizes selected results where we have a high ratio of $\beta / n_{s}$ to give $\mathrm{RCH}$ and $\mathrm{HCH}$ the best chances for success (i.e., for minimizing the fraction of cascades). While $\mathrm{CBH}$ and $\mathrm{PBH}$ halt almost all 100 iterations, $\mathrm{RCH}$ and $\mathrm{HCH}$ allow cascades in $38 \%$ to $100 \%$ of iterations. To obtain the same fraction of cascades as for random and high-degree critical nodes, $\mathrm{CBH}$ would require only about $\beta=5$ critical nodes. Neither $\mathrm{RCH}$ nor $\mathrm{HCH}$ focus on specific seed sets, and $\mathrm{RCH}$ can select nodes of degree 1 as critical (of which there are many in the three networks); these nodes do not propagate complex contagions, so specifying them as critical is wasteful.

Figure 5(a) shows cumulative number of affected nodes as a function of time for the slashdot network with $\mathrm{CBH}$. Results from the 40 iterations that cascade

Table 3. Comparison of $\mathrm{CBH}$ and $\mathrm{PBH}$ against random critical nodes and high-degree critical nodes, with respect to the fraction of iterations in which cascades occur, for $t=2$ and $\beta=500$. Each cell has two entries: one value for $n_{s}=2$ and one for $n_{s}=3$.

\begin{tabular}{|l|c|c|c|c|c|}
\hline Network & $\begin{array}{c}\text { Numbers } \\
\text { of Seeds }\end{array}$ & $\begin{array}{c}\text { Fraction } \\
\text { of Cascades } \\
\text { Random }\end{array}$ & $\begin{array}{c}\text { Fraction } \\
\text { of Cascades } \\
\text { High-Degree }\end{array}$ & $\begin{array}{c}\text { Fraction } \\
\text { of Cascades } \\
\text { CBH }\end{array}$ & $\begin{array}{c}\text { Fraction } \\
\text { of Cascades } \\
\text { PBH }\end{array}$ \\
\hline epinions & $2 / 3$ & $0.94 / 1.00$ & $0.75 / 0.99$ & $0.00 / 0.00$ & $0.00 / 0.01$ \\
\hline wikipedia & $2 / 3$ & $0.96 / 1.00$ & $0.65 / 0.99$ & $0.00 / 0.00$ & $0.00 / 0.01$ \\
\hline slashdot & $2 / 3$ & $0.60 / 0.95$ & $0.38 / 0.80$ & $0.00 / 0.00$ & $0.00 / 0.00$ \\
\hline
\end{tabular}




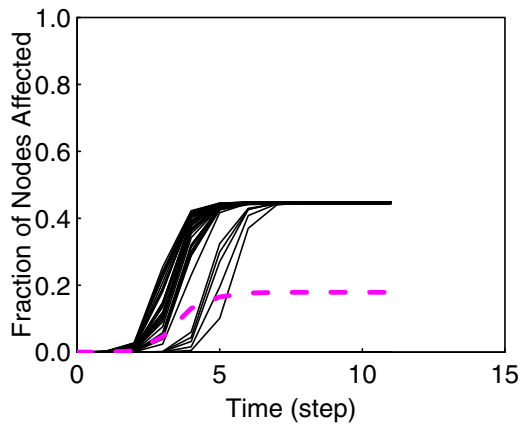

(a)

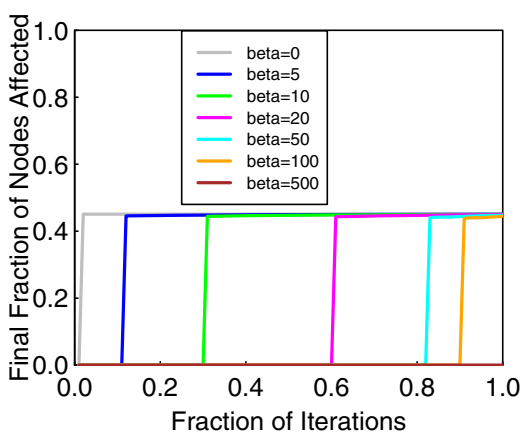

(b)

Fig. 5. (a) Cumulative number of affected nodes for each iteration (solid lines) and average over all 100 iterations (dashed line) for heuristic $\mathrm{CBH}$, for the case $t=3$, $n_{s}=10$, and $\beta=20$ with the slashdot network. (b) Final number of affected nodes for the slashdot network and CBH heuristic for $t=3$ and $n_{s}=10$.

are plotted as solid lines; all iterations plateau at $44 \%$ of the network nodes. (To be precise, the number of nodes affected varies by a very small amount - about $2 \%$ or less - for different sets of seed nodes. Also, in a very few instances, an iteration with no critical nodes also halts the diffusion. We ignore these minor effects throughout for clarity of presentation.) These features are observed in all simulation results and are dictated by the deterministic state transition model: if the diffusion is not halted by the critical nodes, then the size of the outbreak is the same for all iterations, although the progression may vary.

The final fractions of nodes affected for each of the 100 iterations, arranged in increasing numerical order, are plotted as the $\beta=20$ curve in Figure $5(\mathrm{~b})$. The other curves correspond to different budget values, and all exhibit a sharp phase transition, except for $\beta=500$, which halts all iterations. Over all the simulations conducted in this study, both heuristics produce this type of phase transition.

Figure 6 examines the regime of small numbers of seed nodes, and depicts the fraction of iterations that cascade as a function of $\beta$ for two networks. Note the larger discrepancy between heuristics in Figure 6(a) for $\beta=10$; this is explained below. In both plots a $(1 / \beta)$ behavior is observed, so that the number of cascades drops off sharply with increasing budget, but to completely eliminate all cascades in the wikipedia network in Figure 6(a) for example, $\beta=500$ is required for both heuristics when $n_{s}=5$.

Figure 7 provides results that show the greatest differences in the fraction of iterations to cascade for the two heuristics, which generally occur for the largest sizes of seed sets. The results are for the same conditions as in Figure 6(b). For example, in Figure 7, only $17 \%$ of iterations result in a cascade with $\mathrm{CBH}$, while $\mathrm{PBH}$ permits $63 \%$ for $n_{s}=10$.

In all cases, $\mathrm{CBH}$ is at least as effective as $\mathrm{PBH}$. This is because $\mathrm{CBH}$ focuses on conditions at one time step that are required to halt diffusion. $\mathrm{PBH}$, in contrast, can span multiple time steps in that a parent of a high-potential 


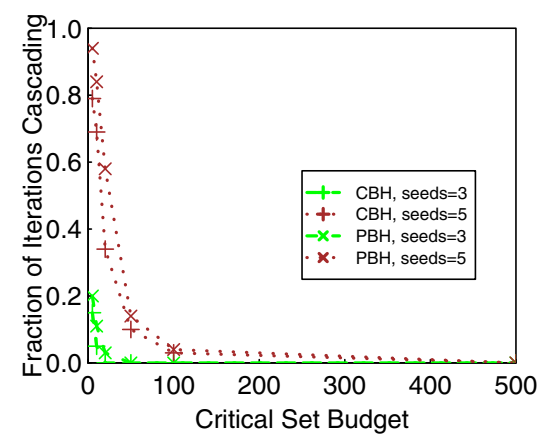

(a)

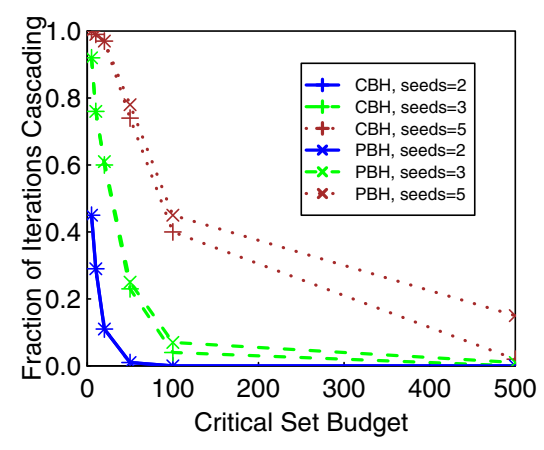

(b)

Fig. 6. Comparisons of $\mathrm{CBH}$ and $\mathrm{PBH}$ in inhibiting diffusion in (a) the wikipedia network for $t=3$; (b) the epinions network for $t=2$.

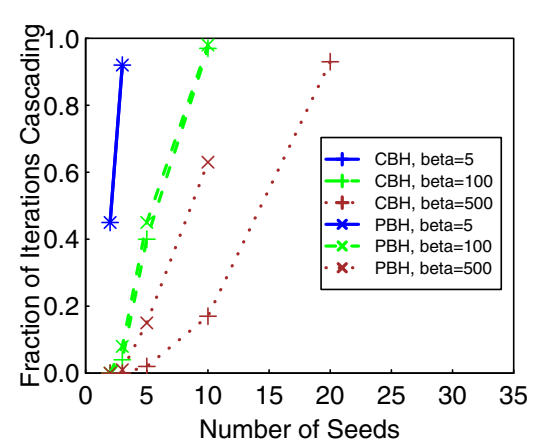

Fig. 7. Comparisons of $\mathrm{CBH}$ and $\mathrm{PBH}$ in inhibiting diffusion in the epinions network for $t=2$

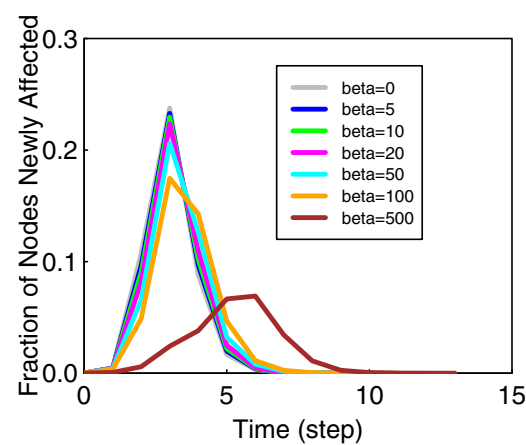

Fig. 8. Average curves of newly affected nodes for PBH for the case $t=2$, $n_{s}=10$, and different values of $\beta$ with the epinions network

node will itself be a high potential node, and hence both may be determined critical. Consequently, there is greater chance for critical nodes to redundantly save salvageable nodes at the expense of others, rendering the critical set less effective. This behavior is the cause of PBH allowing more cascades for $\beta=10$ and $n_{s}=5$ in Figure 6(a)

In Figure 8, the average number of newly affected nodes in each time step over 100 iterations is given for simulations with different numbers of critical nodes. While a budget of $\beta=500$ does not halt the diffusion process, it does slow the diffusion, moving the time of peak number of newly affected nodes from 3 to 6 . This may be useful in providing decision-makers more time for suitable interventions. 


\section{$7 \quad$ Future Work}

There are several directions for future work. Among these are: (a) development of practical heuristics for the critical set problem for complex contagions when there are weights on edges (to model the degree to which a node is influenced by a neighbor); (b) investigation of the critical set problem for complex contagions when the diffusion process is probabilistic; and (c) formulation and study of the problem for time-varying networks in which nodes and edges may appear and disappear over time.

Acknowledgment. We thank the referees from ECML PKDD 2010. We also thank our external collaborators and members of the Network Dynamics and Simulation Science Laboratory (NDSSL) for their suggestions and comments. This work has been partially supported by NSF Nets Grant CNS- 0626964, NSF HSD Grant SES-0729441, NIH MIDAS project 2U01GM070694-7, NSF PetaApps Grant OCI-0904844, DTRA R\&D Grant HDTRA1-0901-0017, DTRA CNIMS Grant HDTRA1-07-C-0113, NSF NETS CNS-0831633, DHS 4112-31805, NSF CNS-0845700 and DOE DE-SC003957.

\section{References}

1. Arulselvan, A., Commander, C.W., Elefteriadou, L., Pardalos, P.M.: Detecting Critical Nodes in Sparse Graphs. Comput. Oper. Res. 36(7), 2193-2200 (2009)

2. Barrett, C.L., Hunt III, H.B., Marathe, M.V., Ravi, S.S., Rosenkrantz, D.J., Stearns, R.E.: Complexity of Reachability Problems for Finite Discrete Dynamical Systems. J. Comput. Syst. Sci. 72(8), 1317-1345 (2006)

3. Borgatti, S.: Identifying sets of key players in a social network. Comput. Math. Organiz. Theor. 12, 21-34 (2006)

4. Centola, D., Eguiluz, V., Macy, M.: Cascade Dynamics of Complex Propagation. Physica A 374, 449-456 (2006)

5. Centola, D., Macy, M.: Complex Contagions and the Weakness of Long Ties. American Journal of Sociology 113(3), 702-734 (2007)

6. Cha, M., Mislove, A., Adams, B., Gummadi, K.: Characterizing Social Cascades in Flickr. In: Proc. of 1st First Workshop on Online Social Networks, pp. 13-18 (2008)

7. Chakrabarti, D., Wang, Y., Wang, C., Leskovec, J., Faloutsos, C.: Epidemic Thresholds in Real Networks. ACM Trans. Inf. Syst. Secur. 10(4), 13-1-13-26 (2008)

8. Dezso, Z., Barabasi, A.: Halting Viruse. In: Scale-Free Networks. Physical Review E 65, 055103-1-055103-4 (2002)

9. Domingos, P., Richardson, M.: Mining the Network Value of Customers. In: Proc. ACM Intl. Conf. on Data Mining and Knowledge Discovery (KDD 2001), pp. 57-61 (2001)

10. Dreyer, P., Roberts, F.: Irreversible $k$-Threshold Processes: Graph-Theoretical Threshold Models of the Spread of Disease and Opinion. Discrete Applied Mathematics 157, 1615-1627 (2009)

11. Easley, D., Kleinberg, J.: Networks, Crowds, and Markets. Cambridge University Press, Cambridge (2010) 
12. Eubank, S., Kumar, V.S.A., Marathe, M.V., Srinivasan, A., Wang, N.: Structure of Social Contact Networks and Their Impact on Epidemics. In: Abello, J., Cormode, G. (eds.) Discrete Methods in Epidemiology. DIMACS Series in Discrete Mathematics and Theoretical Computer Science, pp. 179-200. American Mathematical Society, Providence (2006)

13. Garey, M.R., Johnson, D.S.: Computers and Intractability: A Guide to the Theory of NP-completeness. W. H. Freeman and Co., San Francisco (1979)

14. Granovetter, M.: The Strength of Weak Ties. American Journal of Sociology 78(6), 1360-1380 (1973)

15. Granovetter, M.: Threshold Models of Collective Behavior. American Journal of Sociology 83(6), 1420-1443 (1978)

16. Gruhl, D., Guha, R., Liben-Nowell, D., Tomkins, A.: Information Diffusion Through Blogspace. In: Proc. of the 13th International World Wide Web Conference (WWW 2004), pp. 491-501 (2004)

17. Guha, R., Kumar, R., Raghavan, P., Tomkins, A.: Propagation of Trust and Distrust. In: Proc. of the 13th International World Wide Web Conference (WWW 2004), pp. 403-412 (2004)

18. Habiba, Yu, Y., Berger-Wolf, T., Saia, J.: Finding Spread Blockers in Dynamic Networks. In: The 2nd SNA-KDD Workshop 2008, SNA-KDD 2008 (2008)

19. Harris, K.: The National Longitudinal Study of Adolescent Health (Add Health), Waves I and II, 1994-1996; Wave III, 2001-2002 [machine-readable data file and documentation]. arolina Population Center, University of North Carolina at Chapel Hill, Chapel Hill, NC (2008)

20. Kempe, D., Kleinberg, J., Tardos, E.: Maximizing the Spread of Influence Through a Social Network. In: Proc. ACM Intl. Conf. on Data Mining and Knowledge Discovery (KDD 2003), pp. 137-146 (2003)

21. Kempe, D., Kleinberg, J., Tardos, E.: Influential Nodes in a Diffusion Model for Social Networks. In: Caires, L., Italiano, G.F., Monteiro, L., Palamidessi, C., Yung, M. (eds.) ICALP 2005. LNCS, vol. 3580, pp. 1127-1138. Springer, Heidelberg (2005)

22. Kleinberg, J.: Cascading Behavior in Networks: Algorithmic and Economic Issues. In: Nissan, N., Roughgarden, T., Tardos, E., Vazirani, V. (eds.) Algorithmic Game Theory, ch. 24, pp. 613-632. Cambridge University Press, New York (2007)

23. Kossinets, G., Kleinberg, J., Watts, D.: The Structure of Information Pathways in a Social Communication Network. In: Proc. ACM Intl. Conf. on Data Mining and Knowledge Discovery, KDD 2008 (2008)

24. Kuhlman, C.J., Anil Kumar, V.S., Marathe, M.V., Ravi, S.S., Rosenkrantz, D.J.: Computational Aspects of Ratcheted Discrete Dynamical Systems (April 2010) (under preparation)

25. Kuhlman, C.J., Anil Kumar, V.S., Marathe, M.V., Ravi, S.S., Rosenkrantz, D.J.: NDSSL Technical Report No. 10-060 (2010), http://ndssl.vbi.vt.edu/download/kuhlman/tr-10-60.pdf

26. Leskovec, J., Adamic, L., Huberman, B.: The Dynamics of Viral Marketing. ACM Transactions on the Web, 1(1) (2007)

27. Leskovec, J., Huttenlocher, D., Kleinberg, J.: Predicting Positive and Negative Links in Online Social Networks. In: WWW 2010 (2010)

28. Leskovec, J., Krause, A., Guestrin, C., Faloutsos, C., VanBriesen, J., Glance, N.: Cost-Effective Outbreak Detection in Networks. In: Proc. ACM Intl. Conf. on Data Mining and Knowledge Discovery, KDD 2007 (2007)

29. Leskovec, J., Lang, K., Dasgupta, A., Mahoney, M.: Community Structure in Large Networks: Natural Cluster Sizes and the Absence of Large Well-Defined Clusters (2008), Appears as arXiv.org:0810.1355 
30. Richardson, M., Agrawal, R., Domingos, P.: Trust Management for the Semantic Web. In: Fensel, D., Sycara, K., Mylopoulos, J. (eds.) ISWC 2003. LNCS, vol. 2870, pp. 351-368. Springer, Heidelberg (2003)

31. Richardson, M., Domingos, P.: Mining Knowledge-Sharing Sites for Viral Marketing. In: Proc. ACM Intl. Conf. on Data Mining and Knowledge Discovery (KDD 2002), pp. 61-70 (2002)

32. Tantipathananandh, C., Berger-Wolf, T.Y., Kempe, D.: A Framework for Community Identification in Dynamic Social Networks. In: Proc. ACM Intl. Conf. on Data Mining and Knowledge Discovery (KDD 2007), pp. 717-726 (2007) 